\title{
r-modes in low temperature colour-flavour-locked superconducting quark stars
}

\author{
N. Andersson ${ }^{1}$, B. Haskell ${ }^{1}$ and G.L. Comer ${ }^{2}$ \\ ${ }^{1}$ School of Mathematics, University of Southampton, Southampton, UK \\ ${ }^{2}$ Department of Physics and Center for Fluids at All Scales, Saint Louis University, St Louis, USA
}

(Dated: November 20, 2018)

\begin{abstract}
We present the first multi-fluid analysis of a dense neutron star core with a deconfined colourflavour-locked superconducting quark component. Accounting only for the condensate and (finite temperature) phonons, we make progress by taking over results for superfluid ${ }^{4} \mathrm{He}$. The resultant two-fluid model accounts for a number of additional viscosity coefficients (compared to the NavierStokes equations) and we show how they enter the dissipation analysis for an oscillating star. We provide simple estimates for the gravitational-wave driven $r$-mode instability, demonstrating that the various phonon processes that we consider are not effective damping agents. Even though the results are likely of little direct astrophysical importance (since we consider an overly simplistic stellar model) our analysis represents significant technical progress, laying the foundation for more detailed numerical studies and preparing the ground for the inclusion of additional aspects (in particular associated with kaons) of the problem.
\end{abstract}

\section{INTRODUCTION}

The state of matter at extreme densities continues to be an issue of vigorous investigation. The problem is complicated, not only from the theoretical point of view, but also by the fact that laboratory experiments are restricted. For example, while colliders like RHIC at Brookhaven, GSI in Darmstadt and the LHC at CERN probe hot quark-gluon plasmas they will never be able to explore the cold, extreme high density, region of the QCD phase diagram. In order to test our understanding of the relevant physics we need to turn to astrophysics, and the dynamics of compact stars. In fact, "neutron stars" represent unique laboratories of such extreme physics. With core densities reaching about one order of magnitude beyond nuclear saturation, they are likely to contain exotic states of matter like hyperon phases with net strangeness and/or deconfined quarks. It is well-established that these states of matter should exhibit superfluidity/superconductivity at the relevant temperatures (neutron stars are born with temperatures $\sim 10^{12} \mathrm{~K}$ and rapidly cool below $\sim 10^{9} \mathrm{~K}$ ). Moreover, observed radio pulsar glitches provide strong evidence for the presence of a partially decoupled superfluid component in these systems. The modelling of the dynamics of these, potentially very complex, objects presents a serious challenge.

A key aspect of the problem concerns the fact that a superfluid system has additional dynamical degrees of freedom. This is well-known from experiments on laboratory systems like ${ }^{4} \mathrm{He}$, which exhibit a second sound associated with thermal waves [1, 2]. Analogous "superfluid" modes have been studied in detail for superfluid neutron-proton-electron mixtures relevant for the outer core of a neutron star [3 -5]. For simplicity, these studies have almost exclusively ignored thermal effects (the work in [6] is a notable exception). While this is a useful first approximation it is clear that the zero temperature assumption must be relaxed in a realistic model. Basically, due to the density dependence of the various superfluid pairing gaps (see 7] for a guide to the literature), there will always be regions in a neutron star where thermal effects are important (in the vicinity of the critical density at which the phase transition occurs). Understanding the nature of these transition regions, and their effect on various aspects of neutron star dynamics, is one of the main challenges for research in this area.

In this paper, we describe a first attempt at modelling thermal dynamics in a superfluid neutron star. We focus on stars with a colour-flavour-locked (CFL) superconducting quark core [8] at finite temperatures. This is an interesting problem for several reasons. First of all, the simplest possible model for this system considers a quark condensate coupled to a gas of phonons. This problem is analogous to ${ }^{4} \mathrm{He}$ at low temperatures, and hence we can bring our recent dissipative two-fluid model [9] to bear on it (more or less directly). The lessons we learn from this exercise should inform the development of finite temperature models for the superfluids in the outer core and the neutron star crust. Secondly, even though there have been discussions of the dynamics of the different CFL phases, in particular in the context of the gravitational-wave driven r-mode instability (see [10] for references), the superfluid aspects have (as far as we are aware) not previously been accounted for. Our discussion begins to address the relevance of the additional degrees of freedom in these systems, and provides some insight into the nature of the different "fluids" involved.

As an application with immediate astrophysical relevance, we will work out the inertial r-modes and estimate the relevant viscous damping rates for the "simplest" model of CFL matter. We consider the "cool" regime where the temperature is significantly below all the quasiparticle energy gaps. In this regime, dissipation may mainly occur due to phonon interactions. One reason for considering this model is that there are results in the literature for both bulk- and shear viscosity [11, 12] as well as the mutual friction associated with superfluid vortices [13]. Of 
course, the simple "condensate plus phonon" model that we consider is not the whole story. It should apply at asymptotically high densities, but may not be the true ground state at lower densities. The discussion in [15 17] adds extra dimensions to the problem by considering the bulk viscosity due to kaons (allowing for flavour-changing processes), which will be present at higher temperatures. At first sight, this mechanism will only be relevant for very hot stars $\left(T>1 \mathrm{MeV} \sim 10^{10} \mathrm{~K}\right)$ since it assumes that there is a thermal population of kaons. Below the critical energy where the kaons appear the contribution to the bulk viscosity is exponentially suppressed and may not be that important. However, in more recent work on the so-called CFL-K ${ }^{0}$ phase [16], it is argued that the main low temperature mechanism involves condensed kaons. This is important since the kaon condensate will remain present as $T \rightarrow 0$. The upshot of this is that the problem requires a "multi-fluid" analysis at all temperatures. The simplest model would have three components; the quark condensate, the kaon condensate and finite temperature excitations (phonons and thermal kaons). This problem is more involved than the case that we focus on here. Nevertheless, our results provide an essential starting point for investigations into the dynamical role of the kaons. Most importantly, by providing the "hydrodynamics" view of the problem we illustrate the input needed to study various dynamical scenarios. This should stimulate further discussion between experts on different aspects of this multi-faceted problem, as required to make progress in the future.

\section{FLUX-CONSERVATIVE TWO-FLUID MODEL}

We consider a deconfined quark system system that contains a single CFL superconducting condensate and a phonon gas. Formally, this problem is identical to that for ${ }^{4} \mathrm{He}$ at low temperatures. Hence, we can take as our starting point the recent discussion of superfluid Helium [9], which is based on the convective variational approach to multi-fluid dynamics [18 20] and which we know is in one-to-one correspondence with the orthodox formulation developed by, in particular, Khalatnikov [1].

The variational model takes as its starting point an energy functional $E$, representing the equation of state. This energy determines the relation between the various fluxes and the associated canonical momenta. In the present case, we distinguish between the massive particles in the system, with number density $n$ and flowing with $n v_{i}^{\mathrm{n}}$ from a massless entropy component with number density $s$ and flux $s v_{i}^{\mathrm{s}}$. The latter represents the phonons (which are treated in the fluid approximation, i.e., we assume that there exists a suitable defined "average" transport velocity for each of the two components in the system) The two momentum densities then follow from [51]

$$
\pi_{i}^{\mathrm{n}}=m n v_{i}^{\mathrm{n}}-2 \alpha w_{i}^{\mathrm{ns}},
$$

and

$$
\pi_{i}^{\mathrm{s}}=2 \alpha w_{i}^{\mathrm{ns}},
$$

where $w_{i}^{\mathrm{ns}}=v_{i}^{\mathrm{n}}-v_{i}^{\mathrm{s}}$ and $\alpha$ represents the entropy entrainment 21]. It has been assumed that $E=E\left(n, s, w^{2}\right)$, as expected in an isotropic system, and we have defined

$$
\alpha=\left.\frac{\partial E}{\partial w^{2}}\right|_{n, s}
$$

omitting the indices on $w$ for clarity.

As discussed in [9, 19], the associated momentum equations can be written

$$
f_{i}^{\mathrm{n}}=\partial_{t} \pi_{i}^{\mathrm{n}}+\nabla_{j}\left(v_{\mathrm{n}}^{j} \pi_{i}^{\mathrm{n}}+D_{i}^{\mathrm{n} j}\right)+n \nabla_{i}\left(\mu_{\mathrm{n}}-\frac{1}{2} m v_{\mathrm{n}}^{2}\right)+\pi_{j}^{\mathrm{n}} \nabla_{i} v_{\mathrm{n}}^{j},
$$

and

$$
f_{i}^{\mathrm{s}}=\partial_{t} \pi_{i}^{\mathrm{s}}+\nabla_{j}\left(v_{\mathrm{s}}^{j} \pi_{i}^{\mathrm{s}}+D_{i}^{\mathrm{s} j}\right)+s \nabla_{i} T+\pi_{j}^{\mathrm{s}} \nabla_{i} v_{\mathrm{s}}^{j},
$$

where $\mu_{\mathrm{n}}$ is the matter (quark) chemical potential;

$$
\mu_{\mathrm{n}}=\left(\frac{\partial E}{\partial n}\right)_{s, w^{2}},
$$

and we have used the fact that the temperature corresponds to the entropy chemical potential [21];

$$
T=\mu_{\mathrm{s}}=\left(\frac{\partial E}{\partial s}\right)_{n, w^{2}} .
$$


In these expressions, $D_{i j}^{\mathrm{x}}$ represent the viscous stresses while the "forces" $f_{i}^{\mathrm{x}}$ allow for momentum transfer between the two components. In the following we will assume that the system is isolated, which means that $f_{i}^{\mathrm{n}}+f_{i}^{\mathrm{s}}=0$. As we will discuss later, the force terms can also be used to account for "external" forces like gravity.

In the present context, when we are dealing with a single matter quantity, we will have [19]

$$
\partial_{t} n+\nabla_{j}\left(n v_{\mathrm{n}}^{j}\right)=\Gamma_{\mathrm{n}}=0,
$$

as there is no particle creation/destruction. At the same time the entropy can increase, so we have

$$
\partial_{t} s+\nabla_{j}\left(s v_{\mathrm{s}}^{j}\right)=\Gamma_{\mathrm{s}} \geq 0 .
$$

We also have [19]

$$
T \Gamma_{\mathrm{s}}=-f_{i}^{\mathrm{n}} w_{\mathrm{ns}}^{i}-D_{i}^{j} \nabla_{j} v_{\mathrm{s}}^{i}-D_{i}^{\mathrm{n} j} \nabla_{j} w_{\mathrm{ns}}^{i},
$$

where

$$
D_{i j}=D_{i j}^{\mathrm{n}}+D_{i j}^{\mathrm{s}} .
$$

For an isolated system we know that, if we impose the superfluid constraint of irrotationality the number of dissipation coefficients reduces significantly. As discussed in [9], we have

$$
-D_{i j}=g_{i j}\left(\hat{\zeta}^{\mathrm{n}} \nabla_{l} j^{l}+\zeta \Theta_{\mathrm{s}}\right)+2 \eta \Theta_{i j}^{\mathrm{s}} .
$$

where we have defined $j^{i}=n w_{\mathrm{ns}}^{i}$. We also have;

$$
\frac{1}{n}\left(f_{i}^{\mathrm{n}}-\nabla_{l} D_{i}^{\mathrm{n} l}\right)=\nabla_{i} \Psi
$$

with

$$
\Psi=\hat{\zeta}^{\mathrm{nn}} \nabla_{l} j^{l}+\hat{\zeta}^{\mathrm{n}} \Theta_{\mathrm{s}} .
$$

At this point, only four dissipation coefficients remain in the problem.

In the above expressions, we have used the standard decomposition;

$$
\nabla_{i} v_{j}^{\mathrm{s}}=\Theta_{i j}^{\mathrm{s}}+\frac{1}{3} g_{i j} \Theta_{\mathrm{s}}+\epsilon_{i j k} W_{\mathrm{s}}^{k}
$$

in terms of, the expansion

$$
\Theta_{\mathrm{s}}=\nabla_{j} v_{\mathrm{s}}^{j}
$$

the trace-free shear

$$
\Theta_{i j}^{\mathrm{s}}=\frac{1}{2}\left(\nabla_{i} v_{j}^{\mathrm{s}}+\nabla_{j} v_{i}^{\mathrm{s}}-\frac{2}{3} g_{i j} \Theta_{\mathrm{s}}\right),
$$

and the "vorticity"

$$
W_{\mathrm{s}}^{i}=\frac{1}{4} \epsilon^{i j k}\left(\nabla_{j} v_{k}^{\mathrm{s}}-\nabla_{k} v_{j}^{\mathrm{s}}\right) .
$$

We use analogous expressions for gradients of the relative velocity. The definition of the various quantities should be obvious from the constituent indices.

However, since the system that we consider is not irrotational we need to consider relaxing the assumptions on the dissipation coefficients. This involves making some subtle decisions. In the case of an irrotational flow it is natural to assume that $f_{\mathrm{n}}^{i}=0$. When the superfluid rotates, and vortices are present, the force will not vanish. It is necessary to account for dissipation due to, for example, the scattering of phonons off of the vortex cores. The standard approach to the rotating problem is to add in this "mutual friction" force, keeping the other dissipative terms as in the irrotational case. This strategy ignores a number of dissipative terms that would, at least in principle, be allowed in the equations of motion [9, 19]. The role of these additional terms has not yet, as far as we are aware, been investigated. 
Ignoring the potential relevance of most of the additional dissipation channels in the irrotational case, we simply account for the presence of vortices by i) assuming that (44) represents fluid elements with both a condensate and a smooth-averaged vorticity arising from the vortices, and ii) accounting for the vortex mediated mutual friction by allowing a force [22]

$$
f_{i}^{\mathrm{mf}}=\mathcal{B}^{\prime} \rho_{\mathrm{n}} n_{v} \epsilon_{i j k} \kappa^{j} w_{\mathrm{ns}}^{k}+\mathcal{B} \rho_{\mathrm{n}} n_{v} \epsilon_{i j k} \epsilon^{k l m} \hat{\kappa}^{j} \kappa_{l} w_{m}^{\mathrm{ns}},
$$

to act on the particles (with a balancing force affecting the excitations). Here $n_{v}$ is the vortex area density and the vector $\kappa^{i}=\kappa \hat{\kappa}^{i}$ (the hat represents a unit vector) is aligned with the rotation axis and has magnitude $\kappa=h / 2 m$. Since we will only consider the effect of phonon scattering off of vortices, we expect to be in the weak mutual friction regime where $\mathcal{B}^{\prime} \ll \mathcal{B}$. This means that the first term in the force (19) can be ignored, leaving only the second, dissipative, contribution.

\section{ROTATING EQUILIBRIUM MODELS}

In order to set the stage for the discussion of r-modes, we need to provide a suitable rotating equilibrium configuration. To do this, we note that the two components flow together (there is not heat flux) when the system is in thermal equilibrium. This means that $w_{i}^{\mathrm{ns}}=0$, which implies that $\pi_{i}^{\mathrm{s}}=0$. Thus, it follows from the entropy momentum equation (5) that we must have

$$
s \nabla_{i} T=0 \quad \longrightarrow \quad T=\text { constant } .
$$

This is trivial; if the system is isothermal then there will be no heat flux.

Rewriting the remaining momentum equation (4), making use of the continuity equation, we find (after accounting for the gravitational force as the gradient of the gravitational potential $\Phi$ )

$$
\partial_{t} v_{i}^{\mathrm{n}}+v_{\mathrm{n}}^{j} \nabla_{j} v_{i}^{\mathrm{n}}+\nabla_{i}\left(\tilde{\mu}_{\mathrm{n}}+\Phi\right)=0
$$

where $\tilde{\mu}_{\mathrm{n}}=\mu_{\mathrm{n}} / m$. Using the Lie-derivative along the flow, $\mathcal{L}_{v_{n}}$, we can rewrite this equation as

$$
\partial_{t} v_{i}^{\mathrm{n}}+\mathcal{L}_{v_{n}} v_{i}^{\mathrm{n}}+\nabla_{i}\left(\tilde{\mu}_{\mathrm{n}}+\Phi-\frac{1}{2} v_{\mathrm{n}}^{2}\right)=0
$$

Restricting ourselves to stationary models we have

$$
\partial_{t} v_{i}^{\mathrm{n}}=0
$$

Next, uniform rotation implies

$$
v_{\mathrm{n}}^{i}=\Omega_{\mathrm{n}} \mathbf{e}_{\varphi}^{i},
$$

and, for axisymmetric models, it then follows that

$$
\mathcal{L}_{\varphi} v_{i}^{\mathrm{n}}=0 .
$$

Hence, the required equilibrium models are determined from the usual Bernoulli-type equation

$$
\tilde{\mu}_{\mathrm{n}}+\Phi-\frac{1}{2} v_{\mathrm{n}}^{2}=\text { constant } .
$$

This analysis shows that a rotating configuration can be obtained in the usual way. Once we assume thermal equilibrium, we are dealing with a single-fluid problem.

\section{LINEAR PERTURBATIONS}

Our main aim is to develop the tools required to make quantitative estimates for the r-mode instability in neutron stars with a CFL core. In doing this, we want to account for the fact that CFL matter requires a multi-fluid description. The analysis proceeds in three steps. First we need to formulate the linear perturbation problem for the system. This step is interesting because, as far as we are aware, this is the first time that perturbations of a matter 
plus massless entropy system have been considered in an astrophysical context. The results provide useful insights into finite temperature superfluid dynamics, and should be relevant in a broader context. The second step corresponds to determining the pulsation modes of the system, in this case the r-modes, and finally we need to estimate the damping timescales associated with the different dissipation channels. In the discussion below, we only work out the first of these steps in detail. In order to obtain useful estimates for the r-mode instability we simplify last two steps by considering a uniform density model. This has the advantage that the r-mode solution is simple, and we can evaluate the dissipation rates analytically. In a sense, we do not expect this approximation to be too bad because it is well known that the density profile for a canonical $1.4 M_{\odot} / 10 \mathrm{~km}$ strange star (described by the MIT bag model) is almost flat (see [23]).

Of course, we really need to develop a consistent model for a realistic equation of state. This is essential if we want to study hybrid stars, where the CFL phase is only present in the core. However, the problem is complicated by the fact that the bulk viscosity damping of the r-modes requires the perturbations to be worked out to second order in the slow-rotation approximation 24]. This necessitates a numerical solution, which makes some of the qualitative behaviour of the results less clear. Another complicating factor is the well-known fact that it only makes sense to use a realistic equation of state in a fully general relativistic analysis (see [25] for discussion). The r-modes in single component relativistic stars have been studied [26 29], but there has not yet been any serious analysis of the corresponding multi-fluid problem (although see [30]). A focussed effort in this direction should be encouraged.

\section{A. The non-dissipative problem}

In order to work out the r-mode solutions, it is advantageous to work in a rotating frame. Focussing, for the moment, on the non-dissipative equations we find that, in a frame rotating uniformly with $\Omega^{i}$ we have

$$
\partial_{t} \pi_{i}^{\mathrm{n}}+\nabla_{j}\left(v_{\mathrm{n}}^{j} \pi_{i}^{\mathrm{n}}\right)+n \nabla_{i}\left(\mu_{\mathrm{n}}+m \Phi-\frac{1}{2} m v_{\mathrm{n}}^{2}\right)+\pi_{j}^{\mathrm{n}} \nabla_{i} v_{\mathrm{n}}^{j}+2 \rho \epsilon_{i j k} \Omega^{j} v_{\mathrm{n}}^{k}=0,
$$

and

$$
\partial_{t} \pi_{i}^{\mathrm{s}}+\nabla_{j}\left(v_{\mathrm{s}}^{j} \pi_{i}^{\mathrm{s}}\right)+s \nabla_{i} T+\pi_{j}^{\mathrm{s}} \nabla_{i} v_{\mathrm{s}}^{j}=0,
$$

It is notable that the Coriolis force does not affect the entropy equation (28). This is, however, not surprising. It is well-known that inertial forces are proportional to the mass, and since our entropy component is taken to be massless it should not be affected by the Coriolis force. From a technical point of view, it means that the problem we consider is subtly different from the two-fluid r-mode problem discussed in [31, 32].

Let us now consider perturbations of the rotating equilibrium models discussed in the previous section. Considering Eulerian perturbations (denoted by $\delta$ ) we have, in the rotating frame (using the Cowling approximation $\delta \Phi=0$, and not explicity denoting the velocities as perturbations since they vanish in the background anyway);

$$
\rho \partial_{t} v_{i}^{\mathrm{n}}-2 \alpha \partial_{t} w_{i}^{\mathrm{ns}}+\rho \nabla_{i} \delta \tilde{\mu}_{\mathrm{n}}+2 \rho \epsilon_{i j k} \Omega^{j} v_{\mathrm{n}}^{k}=0,
$$

and

$$
2 \alpha \partial_{t} w_{i}^{\mathrm{ns}}+s \nabla_{i} \delta T=0 .
$$

Adding these we get an equation for the total perturbed momentum

$$
\rho \partial_{t} v_{i}^{\mathrm{n}}+\rho \nabla_{i} \delta \tilde{\mu}_{\mathrm{n}}+s \nabla_{i} \delta T+2 \rho \epsilon_{i j k} \Omega^{j} v_{\mathrm{n}}^{k}=0 .
$$

Noting that the pressure $p$ is defined by (for a co-rotating equilibrium model)

$$
\nabla_{i} p=\rho \nabla_{i} \tilde{\mu}_{\mathrm{n}}+s \nabla_{i} T,
$$

we see that

$$
\nabla_{i} \delta p=\delta \rho \nabla_{i} \tilde{\mu}_{\mathrm{n}}+\rho \nabla_{i} \delta \tilde{\mu}_{\mathrm{n}}+\delta s \nabla_{i} T+s \nabla_{i} \delta T=\frac{\delta \rho}{\rho} \nabla_{i} p+\rho \nabla_{i} \delta \tilde{\mu}_{\mathrm{n}}+s \nabla_{i} \delta T,
$$

since $\nabla_{i} T=0$ in the background. Hence, we have the usual Euler equation

$$
\rho \partial_{t} v_{i}^{\mathrm{n}}+\nabla_{i} \delta p-\frac{\delta \rho}{\rho} \nabla_{i} p+2 \rho \epsilon_{i j k} \Omega^{j} v_{\mathrm{n}}^{k}=0 .
$$


We also have the perturbed continuity equation for the particles

$$
\partial_{t} \delta \rho+\nabla_{i}\left(\rho v_{\mathrm{n}}^{i}\right)=0
$$

and a conservation law for the entropy

$$
\partial_{t} \delta s+\nabla_{i}\left(s v_{\mathrm{s}}^{i}\right)=\partial_{t} \delta s+\nabla_{i}\left(s v_{\mathrm{n}}^{i}\right)-\nabla_{i}\left(s w_{\mathrm{ns}}^{i}\right)=0 .
$$

The entropy is conserved since there is no heat flux in the background ( $\Gamma_{\mathrm{s}}$ is quadratic in the heat flux [21]).

Once we provide an equation of state for matter, we have all relations we need to study the linear dynamics of a non-dissipative finite temperature CFL quark core.

\section{B. Energy integrals and dissipation}

Our main aim is to work out the r-modes and establish the parameter range in which gravitational-wave emission triggers a secular instability. In order to assess the relevance of this instability we need to consider the various dissipative mechanisms that counteract the growth of an unstable mode. The damping due to shear- and bulk viscosity in a two-component system can be estimated using the strategy set out in [31, 32]. That is, we use energy integrals to estimate the various timescales. In essence, the damping timescale $\tau$ associated with any given process is obtained from

$$
\tau \approx 2 E\left[\frac{d E}{d t}\right]^{-1}
$$

where $E$ is the energy associated with the flow and $d E / d t$ is the rate of energy loss due to dissipation. This estimate should be accurate as long as the dissipation is weak enough that it does not affect the flow on a dynamical timescale.

The energy associated with a given perturbation can be obtained from the equations of motion (29) and (30). Multiplying the first equation by $\bar{v}_{\mathrm{n}}^{i}$ and the second by $\bar{v}_{\mathrm{s}}^{i}$ (where the bars denote complex conjugates) and adding, we find that the kinetic energy is given by

$$
E=\frac{1}{2} \int \rho\left(\left|v_{\mathrm{n}}\right|^{2}-\frac{2 \alpha}{\rho}\left|w_{\mathrm{ns}}\right|^{2}\right) d V .
$$

In the case of the r-modes, this is the leading order contribution to the energy. In fact, it turns out that the second term in the bracket is of higher order in the slow-rotation approximation (for the same reasons as in [32]). Hence, the r-mode energy is well approximated by

$$
E \approx \frac{1}{2} \int \rho\left|v_{\mathrm{n}}\right|^{2} d V
$$

In order to evaluate the energy loss due to viscous damping we add the dissipative part of the stress tensor $D_{i j}$ to the equations of motion. The total energy dissipation then follows from the body integral of

$$
-\bar{v}_{\mathrm{n}}^{i} \nabla^{j} D_{i j}^{\mathrm{n}}-\bar{v}_{\mathrm{s}}^{i} \nabla^{j} D_{i j}^{\mathrm{s}} .
$$

After some algebra, this leads to

$$
\frac{d E}{d t}=-\int\left[\eta \bar{\Theta}_{\mathrm{s}}^{i j} \Theta_{i j}^{\mathrm{s}}+\hat{\zeta}^{\mathrm{nn}}|\Theta|^{2}+2 \hat{\zeta}^{\mathrm{n}} \operatorname{Re}\left(\bar{\Theta} \Theta_{\mathrm{s}}\right)+\zeta\left|\Theta_{\mathrm{s}}\right|^{2}\right] d V,
$$

where we have defined

$$
\Theta=\nabla_{i} j^{i}
$$

Recall the relations (16) and (17) and the definition $j^{i}=n w_{\mathrm{ns}}^{i}$.

In order to combine this result with existing results for the viscosity coefficients we need to translate our variables into those of the "orthodox" two-fluid model due to, for example, Khalatnikov [1]. The required translation has been discussed at length in [9], and the key relations that we need are given in the Appendix. Carrying out the comparison, we find that the shear viscosity terms are exactly the same in the two descriptions. Hence, we can use $\eta$ from [12] 
without change. In the case of the bulk viscosity, we find that the two sets of coefficients have different mass scalings, and we identify

$$
\zeta=\zeta_{2} \quad, \quad \hat{\zeta}^{\mathrm{n}}=m \zeta_{1}=m \zeta_{4} \quad, \quad \hat{\zeta}^{\mathrm{nn}}=m^{2} \zeta_{3} .
$$

This means that, when expressed in terms of the standard coefficients, the bulk viscosity dissipation rate becomes

$$
\frac{d E}{d t}=-\int\left[\eta \bar{\Theta}_{\mathrm{s}}^{i j} \Theta_{i j}^{\mathrm{s}}+m^{2} \zeta_{3}|\Theta|^{2}+2 m \zeta_{1} \operatorname{Re}\left(\bar{\Theta} \Theta_{\mathrm{s}}\right)+\zeta_{2}\left|\Theta_{\mathrm{s}}\right|^{2}\right] d V
$$

To conclude, there are three bulk viscosity coefficients rather than the usual single one, and both dynamical degrees of freedom are needed if we want to evaluate the dissipation integrals. Note that the dissipation is expressed in terms of $j^{i}$ and $v_{s}^{i}$, rather than $v_{\mathrm{n}}^{i}$ and $w_{\mathrm{ns}}^{i}$, the variables that we will solve for when we determine the r-modes. It is, of course, straightforward to express (43) in terms of these variables. Unfortunately, the dissipation integrand is then rather messy. We find

$$
\begin{aligned}
\frac{d E}{d t}=-\int\left\{\eta \left[\bar{\Theta}_{\mathrm{n}}^{i j} \Theta_{i j}^{\mathrm{n}}-\right.\right. & \left.2 \operatorname{Re}\left(\bar{\Theta}_{\mathrm{n}}^{i j} \Theta_{i j}^{\mathrm{ns}}\right)+\bar{\Theta}_{\mathrm{ns}}^{i j} \Theta_{i j}^{\mathrm{ns}}\right] \\
& +\zeta_{2}\left|\Theta_{\mathrm{n}}\right|^{2}+\left[\zeta_{2}-2 \rho \zeta_{1}+\rho^{2} \zeta_{3}\right]\left|\Theta_{\mathrm{ns}}\right|^{2}-2\left(\zeta_{2}-\rho \zeta_{1}\right) \operatorname{Re}\left(\bar{\Theta}_{\mathrm{n}} \Theta_{\mathrm{ns}}\right) \\
& \left.+2 \zeta_{1} \operatorname{Re}\left[\bar{\Theta}_{\mathrm{n}}\left(w_{\mathrm{ns}}^{j} \nabla_{j} \rho\right)\right]-2\left(\zeta_{1}-\rho \zeta_{3}\right)\left[\bar{\Theta}_{\mathrm{ns}}\left(w_{\mathrm{ns}}^{j} \nabla_{j} \rho\right)\right]+\zeta_{3}\left|w_{\mathrm{ns}}^{j} \nabla_{j} \rho\right|^{2}\right\} d V .
\end{aligned}
$$

where

$$
\Theta_{\mathrm{n}}=\nabla_{j} v_{\mathrm{n}}^{j} \quad \text { and } \quad \Theta_{\mathrm{ns}}=\nabla_{j} w_{\mathrm{ns}}^{j} .
$$

However, as we will see later, this expression simplifies considerably in the r-mode problem.

Before moving on, it is worth making a general point. It might be tempting to suggest that the terms involving $w_{\mathrm{ns}}^{i}$ in (44) should be less relevant than those involving only $v_{\mathrm{n}}^{i}$. However, without solving for an actual oscillation mode one cannot make this argument precise. In general, the bulk viscosity contributions from terms involving $w_{\mathrm{ns}}^{i}$ cannot be neglected. Since a mode-oscillation typically involves both degrees of freedom (unless the star is unstratified [33, 34]), we need to determine the nature of the oscillations before we make further simplifications.

\section{A SIMPLE MODEL EQUATION OF STATE}

In order to obtain quantitative results for the r-mode instability we need to provide an equation of state. As our main focus is on the two-fluid aspects of the problem (ignored in previous studies), the equation of state must account for the thermal excitations, i.e. the phonons. Moreover, if we want to investigate the relative importance of the different bulk viscosity terms we need to allow for a relative flow at the perturbative level. This is also important if we want to consider the superfluid mutual friction [13]. However, if we insist on the model being truly "realistic" then the problem becomes much more challenging. Hence, we will make a number of simplifying assumptions. This is not only practical, it is also quite reasonable since this is our first attempt at a quantitative analysis. Future work should aim to relax some of our assumptions.

As discussed in Section II, the equation of state that we require takes the form of an energy functional $E=$ $E\left(n, s, w^{2}\right)$. Once this functional is provided, we can work out all the quantities that we need to construct a rotating model and calculate the r-modes. Unfortunately, we do not have an equation of state of the required form. In fact, some of the parameters that we need are usually not considered, essentially since they are not required if one is only interested in equilibrium configurations. In order to make progress we have to build a suitably simple model equation of state. To do this, we assume that the thermal contribution due to the phonons adds to a zero-temperature equation of state for the condensate. This strategy has previously been developed for a relativistic superfluid [35], and builds on the classic expressions for a non-relativistic phonon gas, see [1].

\section{A. The phonon gas}

Let us assume that, at zero temperature matter is described by a barotropic model such that $E(n, s=0)=E_{0}(n)$. Then the chemical potential follows from

$$
\mu=\frac{d E_{0}}{d n}
$$


the pressure is obtained from

$$
d p_{0}=n d \mu
$$

and it is easy to work out the speed of (first) sound;

$$
c_{0}^{2}=\frac{d p_{0}}{d \rho},
$$

Now consider thermal excitations represented by a phonon gas with a linear dispersion relation, with slope $c_{0}$. Working out the thermodynamics of such a gas one can show that its contribution to the pressure, $\psi$, is given by [35]

$$
\psi=\frac{4 \pi^{5}}{45} \frac{(k T)^{4}}{\left(2 \pi \hbar c_{0}\right)^{3}}\left(1-\frac{w^{2}}{c_{0}^{2}}\right)^{-2} .
$$

Here, and in the following, it is to be understood that $w^{2}$ corresponds to $w_{\mathrm{ns}}^{2}$. From this we obtain the entropy density (in the matter frame) via

$$
s=\frac{\partial \psi}{\partial T}
$$

This leads to the explicit result;

$$
s=\frac{4 \psi}{k T}=\frac{16 \pi^{5}}{45}\left(\frac{k T}{2 \pi \hbar c_{0}}\right)^{3}\left(1-\frac{w^{2}}{c_{0}^{2}}\right)^{-2},
$$

which means that the heat capacity is given by

$$
c_{\mathrm{v}}=T \frac{\partial s}{\partial T}=\frac{2 \pi^{2}}{15}\left(\frac{k T}{\hbar c_{0}}\right)^{3}
$$

in agreement with the phonon result in [16].

Finally, one can show that [35] the "normal fluid" density, $\rho_{\mathrm{N}}$, required in the orthodox superfluid formalism (see Appendix) is given by

$$
\rho_{\mathrm{N}}=\frac{16 \pi^{5}}{45} \frac{1}{(2 \pi \hbar)^{3}}\left(\frac{k T}{c_{0}}\right)^{4}\left(1-\frac{w^{2}}{c_{0}^{2}}\right)^{-3} \frac{1}{c_{0}}
$$

This result is consistent with

$$
\rho_{\mathrm{N}}=\frac{4}{3} \frac{E_{\mathrm{ph}}}{c_{0}^{2}}\left(1-\frac{w^{2}}{c_{0}^{2}}\right)^{-3}
$$

where the phonon energy is

$$
E_{\mathrm{ph}}=\frac{4 \pi^{5}}{15}\left(\frac{k T}{2 \pi \hbar c_{0}}\right)^{3} k T .
$$

In our formulation of the problem, we need the entrainment between particles and entropy. As discussed in [9] the relevant entrainment parameter is related to $\rho_{\mathrm{N}}$ according to

$$
\alpha=-\frac{\rho_{\mathrm{N}}}{2}\left(1-\frac{\rho_{\mathrm{N}}}{\rho}\right)^{-1} \approx-\frac{\rho_{\mathrm{N}}}{2}
$$

where the last approximation is accurate in the low temperature limit, when $\rho_{\mathrm{N}} \ll \rho$. This is the regime that we are considering here.

It is worth noting that the above results lead to

$$
\rho_{\mathrm{N}} \approx 29\left(\frac{T}{10^{9} \mathrm{~K}}\right)^{4} \mathrm{~g} / \mathrm{cm}^{3} .
$$


In order to be consistent we need $\rho_{\mathrm{N}} \ll \rho \approx 7 \times 10^{14} \mathrm{~g} / \mathrm{cm}^{3}$ (the average density for a canonical neutron star), which translates into $T \ll 2 \times 10^{12} \mathrm{~K}$. In other words, the model should be valid for all astrophysical neutron stars.

Let us now use the phonon gas results to construct a "complete" model equation of state. That is, we want to deduce a consistent energy functional $E$. This functional should be such that the temperature is obtained from

$$
T=\left.\frac{\partial E}{\partial s}\right|_{n, w^{2}} .
$$

Now, combining (49) with (51) we find that

$$
\left.\frac{\partial E}{\partial s}\right|_{n, w^{2}}=\frac{1}{B} s^{1 / 3}
$$

where

$$
B=\left(\frac{16 \pi^{5}}{45}\right)^{1 / 3} \frac{1}{2 \pi \hbar c_{0}}\left(1-\frac{w^{2}}{c_{0}^{2}}\right)^{-2 / 3} .
$$

Since $c_{0}=c_{0}(n)$ (by definition) we can integrate to get

$$
E=E_{1}\left(n, w^{2}\right)+\frac{3}{4 B} s^{4 / 3}=E_{1}+3 \psi=E_{1}+E_{\mathrm{ph}}\left(1-\frac{w^{2}}{c_{0}^{2}}\right)^{-2} .
$$

We learn that in the limit of a low relative velocity $\left(w \ll c_{0}\right)$ the equation of state is simply given by

$$
E=E_{1}+E_{\mathrm{ph}} .
$$

This result is quite intuitive. Using the fundamental relation of thermodynamics

$$
p+E=n \mu+s T,
$$

it is also straightforward to show that the total pressure is given by

$$
p=p_{0}+\psi,
$$

as one might have expected.

Now that we have the required energy functional, we can determine the entrainment parameter from

$$
\alpha=\left.\frac{\partial E}{\partial w^{2}}\right|_{n, s}
$$

Working this out, we find that (after some algebra)

$$
\alpha=\frac{\partial E_{1}}{\partial w^{2}}-\frac{\rho_{\mathrm{N}}}{2} .
$$

Comparing to (56) we see that the model is consistent with

$$
\frac{\partial E_{1}}{\partial w^{2}}=0
$$

Hence, it is natural to take $E_{1}=E_{0}(n)$, i.e. simply add the thermal phonon energy to the zero-temperature equation of state. The final result is then

$$
E=E_{0}+3 \psi=E_{0}+E_{\mathrm{ph}}\left(1-\frac{w^{2}}{c_{0}^{2}}\right)^{-2}
$$

In order to proceed, we need to provide the zero-temperature equation of state. A "realistic" model should obviously be based on QCD. It is natural [10] to use the MIT bag model. In the case of CFL matter, this leads to (using units where the speed of light is unity, $c=1$ )

$$
p_{0} \approx \frac{3 \mu^{4}}{4 \pi^{2}}-B+\frac{3 \mu^{2}}{4 \pi^{2}}\left(4 \Delta^{2}-m_{s}^{2}\right),
$$


and

$$
\rho \approx \frac{9 \mu^{4}}{4 \pi^{2}}+B-\frac{3 \mu^{2} m_{s}^{2}}{4 \pi^{2}},
$$

where $B$ is the bag constant and $\Delta$ is the pairing gap associated with the CFL condensate.

For simplicity, we will use

$$
p_{0} \approx \frac{1}{3}(\rho-4 B)
$$

in which case the speed of sound is constant;

$$
c_{0}^{2}=\frac{d p_{0}}{d \rho} \approx \frac{1}{3} .
$$

As discussed in [10, 17], this approximation may be quite good, essentially because the two contributions to the last term in (69) almost exactly cancel each other. Later, when we work out the various r-mode dissipation integrals, we will simplify the problem further by assuming that the density is uniform, with a constant speed of sound.

\section{B. Viscosity}

In order to determine the damping timescale for the unstable r-modes, we need to supplement the non-dissipative model with viscous terms. From the discussion in section IIIB we know that we need one shear viscosity coefficient and three bulk viscosity coefficients. In order to account for dissipation associated with the superfluid vortices, we should also consider the mutual friction. Some of the required viscosity coefficients have been discussed in the literature. In particular, the dissipation due to various phonon interactions has been investigated [11 13]. In fact, the availability of these results is one of the key reasons for us focussing on the condensate plus phonon model. Having said that, the available results are incomplete. To what extent this is the case, and how we have dealt with this problem, will be discussed below.

Before we proceed it is worth iterating the point that we are focusing on the phonons because they represent the simplest in a hierarchy of relevant multi-fluid problems. The presence of kaons, either as thermal excitations or a condensate [15, 16], would require us to extend the model to account for additional degrees of freedom. The issues involved are similar to those for a superfluid hyperon core [25], and we expect to consider them in the future.

Let us first consider the phonon shear viscosity. The relevant viscosity coefficient is determined in [12]. We should use (following the discussion above, we are using $c_{0}^{2}=1 / 3$ here)

$$
\eta=2.46 \times 10^{26}\left(\frac{\mu_{q}}{300 \mathrm{MeV}}\right)^{8}\left(\frac{10^{9} \mathrm{~K}}{T}\right)^{5} \mathrm{~g} / \mathrm{cm} \mathrm{s},
$$

where $\mu_{q}$ is the quark chemical potential. We arrive at this result by taking both $\mu_{\mathrm{n}}$ and the quark "mass" $m$ in the fluid model to be equal to the quark chemical potential $\mu_{q}$. The temperature scaling of $\eta$ is the same as in the case of ${ }^{4} \mathrm{He}$, which makes sense given that the involved phonon processes are the same. However, in the context of neutron stars, this model is severely limited. Basically, the "hydrodynamic" treatment of the phonons is no longer valid if their mean-free path is larger than (or comparable to) the size of the system. As in kinetic theory, the mean-free path associated with the shear viscosity follows from (up to a factor of order unity)

$$
\eta \simeq \rho_{\mathrm{N}} c_{0} \lambda .
$$

For the phonon gas model this leads to

$$
\lambda \simeq 5 \times 10^{14}\left(\frac{\mu_{q}}{300 \mathrm{MeV}}\right)^{8}\left(\frac{10^{9} \mathrm{~K}}{T}\right)^{9} \mathrm{~cm} .
$$

In other words, for a $10 \mathrm{~km}$ star the model will not be valid below $\sim 10^{10} \mathrm{~K}$. This is obviously a problem, since mature neutron stars are expected to be significantly colder than this. In essence, we ought to model the phonons as ballistic in the low temperature regime. However, there is some evidence from laboratory experiments on ${ }^{4} \mathrm{He}$ that the fluid model remains relatively accurate also at lower temperatures. Given this, we will use the two-fluid model also in the regime where it is no longer formally valid. Still, in order to describe such systems we need a different 
model for the shear viscosity. In order to make progress we will simply assume that the mean free path is limited by the size of the system. That is, we will use an effective shear viscosity given by

$$
\eta_{\mathrm{eff}} \simeq \rho_{\mathrm{N}} c_{0} R,
$$

where $R$ is the radius of the star. This may seem like a rather drastic assumption, but it has recently been shown [36] that in the case of Helium it leads to results that agree quite well with the observed sound attenuation. In particular, we would have $\eta_{\text {eff }} \sim \rho_{\mathrm{N}} \sim T^{4}$ at low temperatures, which means that the viscosity weakens as the phonon density decreases. This makes (at least qualitative) sense. Explicitly, we get

$$
\eta_{\mathrm{eff}} \approx 5 \times 10^{17}\left(\frac{T}{10^{9} \mathrm{~K}}\right)^{4} \mathrm{~g} / \mathrm{cms}
$$

This model produces a maximum in the viscosity as a function of temperature, a feature that seems quite natural. Having said that, more detailed work on the low-temperature phonon problem is obviously needed in order to improve on our results. This is, in fact, a very interesting problem; when the mean-free path is large, the phonons interact with the "surface" more frequently than with each other. In principle, the hydrodynamics approach should not be valid in this regime. Yet, there is some evidence from studies of heat conduction in nano-systems (see [21] for a recent discussion) that a judicious choice of surface boundary condition for the phonons leads to a useful "fluid" model. We plan to explore this idea further in the future.

We now turn to the bulk viscosity. Only one of the three required coefficients has, so far, been calculated in detail. In the static limit, the analysis in [1] leads to the result

$$
\zeta_{2}(\omega=0)=1.75 \times 10^{12}\left(\frac{m_{s}}{100 \mathrm{MeV}}\right)^{4}\left(\frac{10^{9} \mathrm{~K}}{T}\right) \mathrm{g} / \mathrm{cms},
$$

where $m_{s}$ is the strange quark mass. However, in order to use this result to study oscillation modes, we need consider the frequency dependence in more detail. This is essential since the bulk viscosity is a "resonant" mechanism that is particularly effective when the involved dynamics has a timescale similar to the relevant relaxation time. The static limit only provides partial information. Following [14] we will use

$$
\zeta_{i}=\frac{\tau}{1+\omega^{2} \tau^{2}} \alpha_{i}(T), \quad i=1-3,
$$

where the "amplitudes" $\alpha_{i}$, in general, depend on the different physical scales of the system, while $\tau$ is the relaxation time for the processes that give rise to bulk viscosity. According to [14], the relaxation time scales according to

$$
\tau \sim \frac{c_{0}^{3} \mu_{q}^{8}}{T^{9}}
$$

The scaling of all three viscosity coefficients, in the static limit, has been determined in [14]. We should have

$$
\begin{aligned}
\zeta_{1} & \approx \tilde{\alpha}_{1} \frac{m_{s}^{2}}{T \mu_{q}}, \\
\zeta_{2} & \approx \tilde{\alpha}_{2} \frac{m_{s}^{4}}{T} \\
\zeta_{3} & \approx \tilde{\alpha}_{3} \frac{1}{T \mu_{q}^{2}},
\end{aligned}
$$

where $\tilde{\alpha}_{i}$ are constants. This, together with the relations in (79) and (80) allows us to determine the frequency dependent bulk viscosity coefficients, up to the unknown amplitudes $\tilde{\alpha}_{i}$ and $\beta$. These can not be determined with the approximations used in [14], although we can obviously infer $\tilde{\alpha}_{2}$ from (78). From the analogous problem for ${ }^{4} \mathrm{He}$, we know that the three bulk viscosity coefficients may be of the same order of magnitude. In absence of detailed results for the quark case, it thus makes sense to assume that $\zeta_{1}$ and $\zeta_{3}$ have a similar amplitude to $\zeta_{2}$. In particular, this would suggest that we parametrise $\zeta_{3}$, in the static limit, according to

$$
\zeta_{3}(\omega=0)\left(\frac{\rho}{10^{14} \mathrm{~g} / \mathrm{cm}^{3}}\right)^{2}=\bar{\alpha} \times 10^{12}\left(\frac{10^{9} \mathrm{~K}}{T}\right)\left(\frac{300 \mathrm{MeV}}{\mu_{q}}\right) \mathrm{g} / \mathrm{cms},
$$


where $\bar{\alpha}$ is an unspecified parameter. The frequency dependent result then takes the form

$$
\zeta_{3}=\frac{\zeta_{3}(\omega=0)}{1+\omega^{2} \tau^{2}},
$$

with

$$
\tau=\beta \times 10^{7}\left(\frac{10^{9} \mathrm{~K}}{T}\right)^{9}\left(\frac{\mu_{q}}{300 \mathrm{MeV}}\right)^{8} s .
$$

Here, $\beta$ is another undetermined parameter. In principle, one would expect both $\bar{\alpha}$ and $\beta$ to be of order unity, but given the lack of precise information we can vary them and assess how this affects the bulk viscosity damping of the r-modes. Note that the coefficient in (85) has a maximum at the resonance frequency $\omega=1 / \tau$. We know from previous work that it is essential to understand how this resonance frequency relates to the r-mode frequency. A precise statement to this effect is not possible, given the free parameters in our model, but the strong temperature dependence in (86) means that even a change of several orders of magnitude in $\tau$ would produce a relatively small change in the temperature at which the resonance occurs for a given mode frequency. This suggests that the uncertainty associated with $\beta$ may not affect our analysis too severely (assuming that the temperature scaling is correct, of course). If we, for example, consider a mode frequency $\omega=10^{4} \mathrm{~s}^{-1}$ and an amplitude such that $\tau=10^{8} \mathrm{~s}$, we would have a resonance around $T \approx 3.5 \times 10^{8} \mathrm{~K}$. If, on the other hand, we take a drastically shorter relaxation timescale of $\tau=1 \mathrm{~s}$, then the resonance appears near $T \approx 2.8 \times 10^{9} \mathrm{~K}$. That is, the resonance temperature would shift by less than an order of magnitude. This model is obviously phenomenological, and allows us to proceed, but an actual derivation of the frequency dependent viscosity coefficients is needed if we want more detailed results.

It is obviously important to compare our results to other relevant estimates for the r-modes. The natural, and most immediate, comparison would be to unpaired quark matter. In that case, which corresponds to a single fluid problem, we have [10, 37]

$$
\eta \approx 1.4 \times 10^{17}\left(\frac{0.1}{\alpha_{s}}\right)\left(\frac{\rho}{10^{14} \mathrm{~g} / \mathrm{cm}^{3}}\right)^{5 / 3}\left(\frac{T}{10^{9} \mathrm{~K}}\right)^{-2} \mathrm{~g} / \mathrm{cms}
$$

where $\alpha_{s}$ is the strong interaction coupling constant, and

$$
\zeta=\frac{A(T)}{\omega^{2}+B(T)},
$$

where

$$
A(T)=1.8 \times 10^{36}\left(\frac{\mu_{q}}{300 \mathrm{MeV}}\right)^{3}\left(\frac{m_{s}}{100 \mathrm{MeV}}\right)^{4}\left(\frac{T}{10^{9} \mathrm{~K}}\right)^{2} \mathrm{~g} / \mathrm{cms}^{3}
$$

and

$$
B(T)=2.6 \times 10^{7}\left(\frac{\mu_{q}}{300 \mathrm{MeV}}\right)^{6}\left(1+\frac{m_{s}^{2}}{4 \mu_{q}^{2}}\right)^{2}\left(\frac{T}{10^{9} \mathrm{~K}}\right)^{4} \mathrm{~s}^{-2} .
$$

We also want to quantify the relevance of the vortex mutual friction for the r-mode instability in CFL matter. In order to do this we need a representation of the counter-moving degree of freedom associated with an r-mode (which is of order $\Omega^{2}$ in the slow-rotation approximation) 32]. Before we consider this problem, we need to translate the mutual friction results from [13] to our formalism. In order to relate the parameter $\mathcal{B}$ to the results in [13], let us consider equation (30) where we now include a mutual friction force of the form (19). This leads to an equation for the evolution of the relative velocity of form

$$
\frac{\partial w_{i}^{\mathrm{ns}}}{\partial t}+\frac{s}{2 \alpha} \nabla_{i} \delta T=-\frac{\rho}{2 \alpha}\left[\mathcal{B}^{\prime} n_{v} \epsilon_{i j k} \kappa^{j} w_{\mathrm{ns}}^{k}+\mathcal{B} n_{v} \epsilon_{i j k} \epsilon^{k l m} \hat{\kappa}^{j} \kappa_{l} w_{m}^{\mathrm{ns}}\right] .
$$

Given this equation, and the results in the Appendix, we can compare our formalism to the standard mutual friction description, see e.g. equations (2.2)-(2.3) in [38]. This allows us to determine $\mathcal{B}$ from the parameter $\tilde{\alpha}$ that is calculated in [13]. This leads to

$$
\mathcal{B}=\frac{2 \pi^{5}}{405}\left(1-\frac{2 \alpha}{\rho}\right) \frac{\left(1-c_{0}^{2}\right)^{2}}{c_{0}^{6}}\left(\frac{T}{\mu_{q}}\right)^{5}
$$


or

$$
\mathcal{B} \approx 3.5 \times 10^{-17}\left(1-\frac{2 \alpha}{\rho}\right)\left(\frac{300 \mathrm{MeV}}{\mu_{q}}\right)^{5}\left(\frac{T}{10^{9} \mathrm{~K}}\right)^{5} .
$$

We see that the mutual friction vanishes as the temperature decreases. This obviously makes sense since there will be no phonon-vortex scattering when the phonon gas becomes dilute. Moreover, according to this result, we are safely in the extreme "weak drag" regime (c.f. the discussion in [39]) where

$$
\mathcal{B}^{\prime} \approx \mathcal{B}^{2} \ll 1
$$

This implies that the phonon mutual friction will not damp the r-modes efficiently (probably as expected) [32]. This result is confirmed by the detailed analysis in section VII. However, these results come with an important caveat. Strictly speaking, our analysis is only valid as long as the phonons can be described as a fluid. As we have already explained, this is not the case for mature neutron stars. In fact, the results of [13] are supposedly derived for ballistic phonons. This means that our analysis is somewhat inconsistent. At temperatures above (say) $10^{10} \mathrm{~K}$ the mutual friction parameter from [13] may not apply, and below this temperature our two-fluid model may not be appropriate. However, the latter issue may not be that important. After all, one would expect the phonon mutual friction to weaken dramatically at lower temperature, c.f. (93), meaning the any quantitative errors in the analysis will be of no real importance.

\section{APPROXIMATE R-MODE RESULTS, SHEAR- AND BULK VISCOSITY}

Our (simple) model for a two-component cool CFL core is now "complete", and we may conduct the r-mode analysis as in 31, 32]. In order to quantify the damping due to bulk viscosity, we need to account for terms of order $\Omega^{2}$ in the analysis. This complicates the problem, since the centrifugal deformation of the star's shape enters at the same level. In view of this, and the fact that this is a first exploratory study, we will make a sequence of approximations that allow us to proceed analytically. This strategy also makes sense since we are using a simplified equation of state. The results of our analysis should help determine whether a full numerical study is worthwhile.

First of all, we need to make a choice of primary variables. The linearised equations link the four scalar variables $\delta p, \delta T, \delta s$ and $\delta \rho$. In principle, we can use the equation of state to express any two of these in terms of the other two. Following the strategy set out in [32] we will work with $\delta p$ and $\delta T$. For our model equation of state we have (since the background is co-rotating)

$$
\delta p=\delta p_{0}+\left(\frac{\partial \psi}{\partial T}\right) \delta T=\delta p_{0}+s \delta T
$$

which means that we can express the equations in terms of $\delta p_{0}$ and $\delta T$. We then have

$$
\delta \rho=\left(\frac{\partial \rho}{\partial p_{0}}\right) \delta p_{0}+\left(\frac{\partial \rho}{\partial T}\right) \delta T=\frac{1}{c_{0}^{2}} \delta p_{0}
$$

since $c_{0}$ is contant, and

$$
\delta s=\left(\frac{\partial s}{\partial p_{0}}\right) \delta p_{0}+\left(\frac{\partial s}{\partial T}\right) \delta T=\frac{c_{\mathrm{v}}}{T} \delta T
$$

since $p_{0}=p_{0}(n)$.

In order to avoid numerics we will make use of a "trick" used by Lindblom, Owen and Morsink in one of the early r-mode instability papers [40]. The basic idea is to neglect the rotational change in shape in different terms in the perturbation equations. Once this is done one can "estimate" the bulk viscosity from the leading order r-mode solution. Although this simplification is not not based on a rigorous argument, one can show that the bulk viscosity damping timescale is of the right order of magnitude (within about a factor of 5 of the true second-order slow-rotation result [41]). This is good enough for our present purposes.

In our case, the calculation would proceed as follows: First consider the continuity equation

$$
i \omega \delta \rho+\rho \nabla_{i} v_{\mathrm{n}}^{i}+v_{\mathrm{n}}^{i} \nabla_{i} \rho=0,
$$


where we have assumed that all perturbed quantities behave as $e^{i(\omega t+m \varphi)}$. If we simply omit the last term (that would vanish for a spherical star since an r-mode is purely toroidal to leading order), we get

$$
\nabla_{i} v_{\mathrm{n}}^{i} \approx-\frac{i \omega}{\rho} \delta \rho=-\frac{i \omega}{\rho c_{0}^{2}} \delta p_{0} .
$$

This may not be a very accurate estimate, but it allows us to progress without having to determine the rotational corrections to the r-mode (which depend on the term that we have neglected). Now consider the second degree of freedom in a similar way. From the entropy equation we get

$$
i \omega \delta s+s\left(\nabla_{i} v_{n}^{i}-\nabla_{i} w_{\mathrm{ns}}^{i}\right)+\left(v_{\mathrm{n}}^{i}-w_{\mathrm{ns}}^{i}\right) \nabla_{i} s=0 .
$$

In fact, for the model equation of state the last term vanishes identically since $s=s(T)$ and $\nabla_{i} T=0$ for our background model. It follows that

$$
\nabla_{i} v_{\mathrm{n}}^{i}-\nabla_{i} w_{\mathrm{ns}}^{i} \approx-i \omega \frac{\delta s}{s} .
$$

The approximation is completed by the $\varphi$-components of the two Euler equations. Assuming that the r-mode velocity field takes the form

$$
v_{\mathrm{n}}^{i}=\left[v_{r} \mathbf{e}_{r}^{i}+v_{\theta} \mathbf{e}_{\theta}^{i}+v_{\varphi} \mathbf{e}_{\varphi}^{i}\right] e^{i(\omega t+m \varphi)},
$$

and that $w_{\mathrm{ns}}^{i}$ is similar, although of higher slow-rotation order than $v_{\mathrm{n}}^{i}$ [32], we get

$$
i \omega \rho r^{2} \sin ^{2} \theta v_{\varphi}+i m \delta p+2 \rho \Omega r^{2} \sin \theta \cos \theta v_{\theta}=0,
$$

and

$$
2 i \alpha \omega r^{2} \sin ^{2} \theta w_{\varphi}+i m s \delta T=0 .
$$

The second relation shows immediately that $\delta T$ is of higher slow-rotation order for the classic r-mode (since $w_{\varphi}$ is of higher order and $\omega \sim \Omega$ for inertial modes). This means that we can justifiably neglect the temperature variation in the pressure term in the first equation. That is, we have

$$
i \omega \rho r^{2} \sin ^{2} \theta v_{\varphi}+i m \delta p_{0}+2 \rho \Omega r^{2} \sin \theta \cos \theta v_{\theta}=0 .
$$

This is exactly the result used in [40]. Moreover, we now see that the first term in (100) should also be of higher order, which means that we are left with

$$
\nabla_{i} w_{\mathrm{ns}}^{i} \approx \nabla_{i} v_{\mathrm{n}}^{i} .
$$

The above relations allow us to estimate the terms needed to evaluate the bulk viscosity integrals once we have the leading order r-mode solution. Since the required contribution to the r-mode velocity field is purely toroidal, we have

$$
v_{\theta}=-\frac{U_{l}}{r^{2} \sin \theta} \partial_{\varphi} Y_{l}^{m}, \quad v_{\varphi}=\frac{U_{l}}{r^{2} \sin \theta} \partial_{\theta} Y_{l}^{m} .
$$

Moreover, the mode-solution is such that the only contribution comes from $l=m$ in which case $U_{m}=(r / R)^{m+1}$ (noting that the normalisation is irrelevant at the linear perturbation level). Finally, to the accuracy needed, the mode frequency is (in the rotating frame)

$$
\omega=\frac{2 \Omega}{m+1} .
$$

Using these results, and expanding the (scalar) pressure perturbation in spherical harmonics, i.e. using $\delta p_{0}=$ $\sum_{l} \delta p_{l} Y_{l}^{m} e^{i(\omega t+m \varphi)}$, we find that (105) leads to

$$
\frac{\delta p_{m+1}}{\rho}=\frac{1}{\sqrt{2 m+3}} \frac{2 m \Omega}{m+1} U_{m},
$$

and thus we have

$$
\nabla_{i} v_{\mathrm{n}}^{i} \approx \nabla_{i} w_{\mathrm{ns}}^{i} \approx-\frac{i}{\sqrt{2 m+3}} \frac{4 m \Omega^{2}}{(m+1)^{2} c_{0}^{2}} U_{m} Y_{m+1}^{m} .
$$

This completes the approximation scheme, which provides all the expressions we need to make a rough estimate of the bulk viscosity damping timescale. 


\section{MUTUAL FRICTION}

In order to estimate the mutual friction damping, we need to determine the detailed counter-moving degree of freedom associated with the r-modes. In principle, this calculation proceeds as in [32] and involves the rotational corrections to the shape of the star. However, in order to be consistent we will instead build on the approximations introduced in the previous section. First of all, it is easy to show that the countermoving degree of freedom will be poloidal (in contrast to the toroidal leading order r-mode solution). This means that we have

$$
w_{r}=\frac{1}{r} W_{l} Y_{l}^{m}, \quad w_{\theta}=\frac{V_{l}}{r^{2}} \partial_{\theta} Y_{l}^{m}, \quad w_{\varphi}=\frac{V_{l}}{r^{2} \sin \theta} \partial_{\varphi} Y_{l}^{m} .
$$

If we also expand the temperature perturbation in such a way that $\delta T=\sum_{l} T_{l} Y_{l}^{m}$, then it follows from (104) that

$$
V_{l}=\frac{i s}{2 \alpha \omega} T_{l}
$$

Similarly, we get from the radial component of (30);

$$
W_{l}=\frac{i s}{2 \alpha \omega} \partial_{r} T_{l} .
$$

Combining these results with (108) and (110), we find that

$$
s T_{m+1}=\frac{8 m \alpha \Omega^{3}}{\sqrt{2 m+3}(m+1)^{3}(2 m+5)} \frac{R^{2}}{c_{0}^{2}}\left(\frac{r}{R}\right)^{m+1}\left[\frac{m+3}{m+1}-\left(\frac{r}{R}\right)^{2}\right] .
$$

From this result we easily obtain the required counter-moving components of the r-mode.

We arrive at (114) by assuming that $w_{r}=0$ at the surface (in accordance with the discussion in [32]). This condition is unlikely to be correct in the present problem, and a detailed analysis of the appropriate condition to impose on the phonons needs to be carried out in the future (c.f. the discussion in 21]). At this point we simply note that our assumption that the whole star is composed of CFL matter is artificial anyway. Moreover, the boundary condition does not affect the parameter scaling of the result. This is the dominant factor in determining the r-mode damping timescale.

\section{THE R-MODE INSTABILITY WINDOW}

To estimate the growth/damping timescales for the r-mode instability we need to evaluate various energy integrals, see [24, 40]. First of all, the mode energy is given by

$$
E=\frac{1}{2} m(m+1) R^{-(2 m+2)} \int_{0}^{R} \rho r^{2 m+2} d r,
$$

while the growth time-scale due to (current multipole) gravitational-wave emission is obtained from

$$
\frac{1}{\tau_{\mathrm{gw}}}=-\frac{32 \pi G \Omega^{2 m+2}}{c^{2 m+3}} \frac{(m-1)^{2 m}}{[(2 m+1) ! !]^{2}}\left(\frac{m+2}{m+1}\right)^{2 m+2} \int_{0}^{R} \rho r^{2 m+2} d r .
$$

Turning to the shear viscosity, we know that $\left|w_{\mathrm{ns}}\right| \ll\left|v_{\mathrm{n}}\right|$ for the superfluid r-mode. This means that the shear viscosity integral simplifies to the usual, single fluid result. Hence, the leading-order energy loss due to shear viscosity follows from the expression

$$
\begin{aligned}
\dot{E}_{\mathrm{sv}}=-m(m+1)\left\{\int_{0}^{R} \frac{\eta}{r^{2}}\left[r^{6}\left|\partial_{r}\left(\frac{U_{m}}{r^{2}}\right)\right|^{2}+(m-1)(m+2)\left|U_{m}\right|^{2}\right]\right\} d r & \\
& =-m\left(m^{2}-1\right)(2 m+1) R^{-(2 m+2)} \int_{0}^{R} \eta r^{2 m} d r
\end{aligned}
$$

leading to

$$
\frac{1}{\tau_{\mathrm{sv}}}=-(m-1)(2 m+1) \int_{0}^{R} \eta r^{2 m} d r\left[\int_{0}^{R} \rho r^{2 m+2} d r\right]^{-1}
$$


Next, we need to estimate the bulk viscosity damping. From (44) we know that the general bulk viscosity expression is complicated, especially since we cannot rule out the possibility that the three different bulk viscosity contributions are of similar magnitude. However, the approximations that we have made simplify the problem considerably. Neglecting the terms that arise due to the change in shape of the rotating star [the last line in [44]), and making use of (106) we find that

$$
\dot{E}_{\mathrm{bv}} \approx-\int_{0}^{R} \zeta_{\mathrm{eff}}\left|\nabla_{i} v_{\mathrm{n}}^{i}\right|^{2} d V \approx-\frac{16 m^{2} \Omega^{4}}{(m+1)^{4}(2 m+3)} R^{-(2 m+2)} \int_{0}^{R} \frac{\zeta_{\mathrm{eff}}}{c_{0}^{4}} r^{2 m+4} d r
$$

which leads to

$$
\frac{1}{\tau_{\mathrm{bv}}}=-\frac{16 m \Omega^{4}}{(m+1)^{5}(2 m+3)} \int_{0}^{R} \frac{\zeta_{\mathrm{eff}}}{c_{0}^{4}} r^{2 m+4} d r\left[\int_{0}^{R} \rho r^{2 m+2} d r\right]^{-1}
$$

where

$$
\zeta_{\mathrm{eff}} \approx \rho^{2} \zeta_{3}
$$

This result follows after some surprising cancellations, associated with (106). It is notable that only one of the three bulk viscosity coefficients plays a role in our simplified case. Moreover, it is not the one $\left(\zeta_{2}\right)$ that remains in the Navier-Stokes limit. Of course, one would not expect this drastic simplification for a more realistic stellar model. This fact provides strong motivation for a more detailed, numerical, r-mode analysis. We also learn that we can not, in general, ignore the additional bulk viscosities in the multi-fluid problem.

Finally, we have the dissipation integral for the mutual friction. From the results discussed in [31, 32], we find that

$$
\dot{E}_{\mathrm{mf}} \approx-2 \int \rho \mathcal{B} \Omega\left(\delta_{i}^{m}-\hat{\Omega}^{m} \hat{\Omega}_{i}\right) w_{\mathrm{ns}}^{i} \bar{w}_{m}^{\mathrm{ns}} d V .
$$

Working out the angular integrals, this reduces to

$$
\dot{E}_{\mathrm{mf}} \approx-2 \int \rho \mathcal{B} \Omega\left\{\left[1-Q_{m+2}^{2}-Q_{m+1}^{2}\right] W_{m+1}^{2}+\left[(m+1)(m+2)-(m+1)^{2} Q_{m+2}^{2}-(m+2)^{2} Q_{m+1}^{2}\right] V_{m+1}^{2}\right\} d r
$$

where we have used

$$
Q_{l}^{2}=\frac{(l+m)(l-m)}{(2 l-1)(2 l+1)}
$$

The integral in (123) is easily evaluated using the eigenfunctions from the previous section.

In order to evaluate the relevant integrals, and obtain estimated timescales with explicit parameter scaling, we now assume that the density is uniform. Taking all parameters constant, we easily find that

$$
\tau_{\mathrm{gw}}=-22\left(\frac{1.4 M_{\odot}}{M}\right)\left(\frac{10 \mathrm{~km}}{R}\right)^{4}\left(\frac{P}{1 \mathrm{~ms}}\right)^{6} \mathrm{~s}
$$

for the $l=m=2 \mathrm{r}$-mode (the sign indicates that the mode is unstable). We also get, for the shear viscosity,

$$
\frac{1}{\tau_{\mathrm{sv}}} \approx 1.1 \times 10^{-26}\left(\frac{\eta}{1 \mathrm{~g} / \mathrm{cms}}\right)\left(\frac{R}{10 \mathrm{~km}}\right)\left(\frac{1.4 M_{\odot}}{M}\right) \mathrm{s}^{-1}
$$

and for the bulk viscosity we find

$$
\frac{1}{\tau_{\mathrm{bv}}} \approx 3.8 \times 10^{-31}\left(\frac{\zeta_{\mathrm{eff}}}{1 \mathrm{~g} / \mathrm{cms}}\right)\left(\frac{R}{10 \mathrm{~km}}\right)^{5}\left(\frac{1.4 M_{\odot}}{M}\right)\left(\frac{1 \mathrm{~ms}}{P}\right)^{4} \mathrm{~s}^{-1} .
$$

Finally, in the case of the mutual friction we find

$$
\frac{1}{\tau_{\mathrm{mf}}} \approx 0.17 \mathcal{B}\left(\frac{R}{10 \mathrm{~km}}\right)^{4}\left(\frac{P}{1 \mathrm{~ms}}\right)^{-5} \mathrm{~s}^{-1}
$$




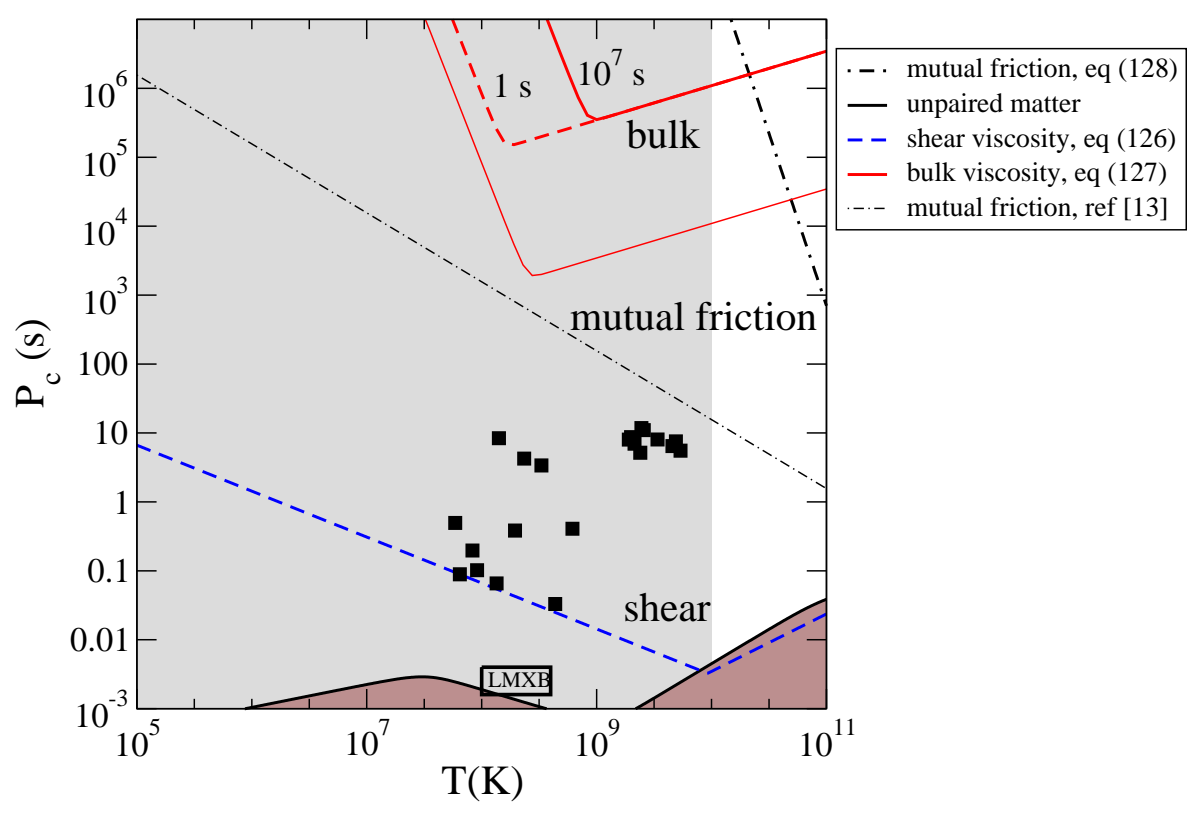

FIG. 1: The r-mode instability window for a dense core comprising a CFL superconducting quark condensate and (finite temperature) phonons. The figure shows the critical rotational period vs core temperature for the instability. Gravitational waves drive the $l=m=2$ r-mode unstable for systems located below the different curves. The effect of shear- and bulk viscosity associated with phonon processes are shown as blue (dashed) and red (solid/dashed) curves, respectively. We show the result for both the canonical value $\beta=1$ (solid red line) and the rather extreme value $\beta=10^{-7}$ (dashed red line) in (866), leading to relaxation times of $10^{7} \mathrm{~s}$ and $1 \mathrm{~s}$ (as indicated in the figure), respectively. We also show the result for the case when $\bar{\alpha}$ is increased by a factor of $10^{4}$ (thin solid red line). Our result for the mutual friction damping (this dash-dot black line) is compared to the result from [13] (thin dash-dot black line), demonstrating the dramatic effect of the different scaling with the rotation. For comparison, we also provide the instability curve for the combined shear- and bulk viscosity, obtained from (87) and (88), in the case of unpaired quark matter (black line at bottom of figure). Finally, we show the observed spin period and inferred core temperature for a number of astrophysical systems (filled squares), and we also indicate the region of parameter space where accreting neutron stars in low-mass X-ray binaries (LMXB) are thought to be located. The region below $10^{10} \mathrm{~K}$, where the phonon-fluid model may not be appropriate, is shown with grey background to emphasize the need for improved modelling in the astrophysically relevant part of parameter space.

This result differs significantly from the estimate in [13], in particular, in terms of the scaling with the rotation rate. However, our result is obtained from the actual counter-moving part of the r-mode solution and the final scaling accords with the standard result (see, for example, [32]). The main difference between our result and that in [13] can be explained by noting that, if we take the r-mode energy to be proportional to $w_{\mathrm{ns}}^{2}$ rather than $v_{\mathrm{n}}^{2}$ then we arrive at a mutual friction damping time scale very similar to that obtained in [13]. The large discrepancy between the estimates thus follows from the fact that the velocites $v_{\mathrm{n}}^{i}$ and $w_{\mathrm{ns}}^{i}$ associated with the r-mode solution are of different orders of $\Omega$ in the slow-rotation approximation.

Combining these estimates with (73), (77), (84) and (93), i.e. balancing the four timescales (125)-(128), we arrive at the r-mode instability window in Figure 1 The figure shows the critical rotational period for the instability as function of the core temperature for a canonical star with mass $1.4 M_{\odot}$ and radius $10 \mathrm{~km}$. Because of the less efficient damping mechanisms, we show the period rather than the rotation rate. This makes sense because the long timescales involved beome much clearer than when the critical rotation rate is expressed as a small fraction of the rotational break-up limit. As a result, gravitational waves drive the $l=m=2 \mathrm{r}$-mode unstable for systems located below the different curves in the figure. Our estimates show that the bulk viscosity is much weaker than the shear viscosity at all temperatures of interest. In order to show how the bulk viscosity result depends on the relaxation time, c.f. (86), we show the result for both the canonical value $\beta=1$ and the rather extreme value $\beta=10^{-7}$, leading to relaxation times of $10^{7} \mathrm{~s}$ and $1 \mathrm{~s}$, respectively. The fact that the associated instability curves do not differ much shows that the results are not very sensitive to this parameter. This is due to the strong scaling with temperature. In order to explore the importance of the overall strength of the bulk viscosity, we also show the result for the case when $\bar{\alpha}$ is increased by a factor of $10^{4}$ compared to our canonical value $\bar{\alpha}=1$. This result shows that the bulk viscosity has to be vastly different from our assumed model in order to be relevant. The data illustrated in figure 1 also shows that the instability curve obtained from balancing the gravitational-wave driving and our result for the phonon mutual friction damping is vastly different from the result in [13]. The different dependence on rotation is obvious, and it is 
clear that the phonon mutual friction would be irrelevant for all astrophysical stars with CFL cores. For comparison, we show the instability curve for the combined shear- and bulk viscosity, obtained using (87) and (88), in the case of unpaired quark matter. It is interesting to note that the phonon shear viscosity in the CFL case leads to the strongest r-mode damping at temperatures above $10^{10} \mathrm{~K}$. Of course, astrophysical neutron stars will cool to temperatures much lower than this soon after birth so this region may not be that relevant. The results in the figure show, quite clearly, that we need to improve our understanding of the low-temperature regime. The instability curve below the $10^{10} \mathrm{~K}$ is obtained using our phenomenological model (77). Future work needs to model this regime in detail.

For comparison, we also show the observed spin and inferred core temperature for a number of astrophysical systems in Figure 1 (the data is taken from [42]). This comparison is somewhat inconsistent because we have assumed that the core is shielded by a normal crust with a thickness of, at least, a few hundred meters (so that the standard heat-blanket argument [43] applies. The presence of a normal matter outer region should provide addition r-mode dissipation channels that we have not considered. In particular, one would expect a viscous boundary layer at the crust-core interface to be a more efficient damping agent (see [4, 45] for the most recent discussion) than the phonon mechanisms that we have considered. We also indicate the region of parameter space where accreting neutron stars in low-mass X-ray binaries would be located (given the usual arguments [46, 47]). These systems would be at variance with the pure CFL plus phonon model in the sense that they would be located deep into the unstable region. If there were an active r-mode instability in these systems, one would expect it to have observational effects on, for example, the spin evolution. Observations do not provide any evidence of this.

\section{CONCLUDING REMARKS}

We have presented the first true multi-fluid analysis of a dense neutron star core with a deconfined, colour-flavourlocked superconducting, quark core. By focussing on a cool system, and accounting only for the condensate and (finite temperature) phonons, we made progress by taking over much of the formalism from the analogous problem for superfluid ${ }^{4} \mathrm{He}$, the archetypal two-fluid laboratory system. The additional fluid degree of freedom, in the present case represented by the phonon gas, leads to the system not being well represented by the Navier-Stokes equations. In particular, a complete model requires a number of additional viscosity coefficients.

Without an actual calculation it is not easy to establish whether the multi-fluid aspects are relevant or not. For example, in the case of the gravitational-wave driven instability of the f-modes it is known that the superfluid degree of freedom is very important, since the vortex mutual friction may completely suppress that instability below a critical temperature (see [31, 48]). It is known that, because of the different nature of the associated velocity field, the mutual friction does not affect the r-mode instability in the same drastic fashion [32, 49]. These examples provide clear evidence that different problems need to be considered on a case-by-case basis.

We have provided a detailed dissipative formulation for a system comprising a quark (CFL) condensate and phonons. The model builds on recent improvements in our understanding of the analogous problems of superfluid ${ }^{4} \mathrm{He}$ [9] and causal heat conductivity [21]. A key ingredient is the massless entropy component that represents the phonon excitations. We have discussed how the superfluid constraint of irrotationality reduces the number of required viscosity coefficients to four (one shear and three bulk), and provided a translation between results in the literature [11, 12] and the coefficients in our formalism. We also emphasised that many more dissipative channels may come into play in a rotating system where superfluid vortices are present [9, 19]. In particular, we showed how the vortex mediated mutual friction is accounted for in the model, and translated the available results for the associated coefficients [13].

In order to be able to make relevant estimates for the r-mode instability, we developed a simple two-component equation of state based on the MIT bag model at zero temperatures with an additional phonon gas representing the thermal component. This example highlights the additional information that is needed in a multi-fluid analysis, in particular, regarding the entrainment coupling. Future work needs to provide a consistent equation of state, including all the key aspects. The model equation of state completed the formulation of the problem and we could, in principle, have carried out a numerical study of the r-modes. We opted not to do this, instead introducing a sequence of simplifying assumptions, because we felt that it would be useful to start by working out some less precise estimates for the relevant dissipation timescales. A numerical analysis of the problem should, of course, be encouraged. The problem has a number of interesting aspects, and may shed light on how one should deal with finite temperature superfluid neutron stars in general.

This work was motivated by a desire to understand the different phases of CFL matter from a hydrodynamics point of view. It is, obviously, an interesting problem and the notion that observations of gravitational waves from relativistic stars may help shed light on the extreme QCD phase diagram is exciting. The r-mode instability has been discussed in this context for some time, see for example [1] 13, 15, 16, 37, 50], but there has not been any previous discussion of the multi-fluid aspects of the problem. We hope that this work will stimulate a more detailed discussion between experts in the relevant areas. 
The fact that the various phonon processes that we have accounted for can be shown to have little effect on the r-mode instability should not discourage future efforts. In fact, the result could probably have been anticipated. The real challenge will be to account for additional degrees of freedom, especially associated with the kaons (either thermal or in a condensate) [15, 16], and the modelling of "hybrid" stars with quark cores and various phase transitions. These problems have additional features that, while possibly understood in principle, have never been considered in practice. This makes the modelling more complex, but also intriguing since the richer dynamics may lead to surprises.

\section{Appendix: Translation to the orthodox framework}

The relationship between the flux conservative formulation and the orthodox framework for superfluid Helium has been examined in detail elsewhere [9, 18]. Still, it is useful to summarise the main results that are needed to relate the different dissipation coefficients. First of all, we identify the "normal" fluid in the standard description with the gas of excitations, which is directly associated with the entropy of the system. This leads to

$$
v_{\mathrm{N}}^{i}=v_{\mathrm{s}}^{i} .
$$

Secondly, the so-called "superfluid" velocity is given by

$$
v_{\mathrm{S}}^{i}=\frac{\pi_{\mathrm{n}}^{i}}{\rho}=(1-\varepsilon) v_{\mathrm{n}}^{i}+\varepsilon v_{\mathrm{s}}^{i},
$$

where $\varepsilon=\frac{2 \alpha}{\rho}$ is the entropy entrainment parameter. This means that the total mass flux takes the form

$$
\rho v_{\mathrm{n}}^{i}=\rho_{\mathrm{N}} v_{N}^{i}+\rho_{\mathrm{S}} v_{\mathrm{S}}^{i}
$$

from which we learn that

$$
\rho_{\mathrm{S}}=\frac{\rho}{1-\varepsilon} \quad \text { and } \quad \rho_{\mathrm{N}}=-\frac{\varepsilon \rho}{1-\varepsilon},
$$

where $\rho_{\mathrm{S}}$ and $\rho_{\mathrm{N}}$ are the superfluid and normal fluid densities.

\section{Acknowledgments}

We are grateful to Mark Alford and Cristina Manuel for a number of useful discussions. NA and BH acknowledge support from STFC via grant number PP/E001025/1. BH also acknowledges support from the European Science Foundation (ESF) for the activity entitled "The New Physics of Compact Stars" , under exchange grant 2449, and thanks the Dipartimento di Fisica, Università degli studi di Milano for kind hospitality during part of this work.

[1] I.M. Khalatnikov, An introduction to the theory of superfluidity (W. A. Benjamin, Inc., New York, 1965).

[2] S.J. Putterman, Superfluid hydrodynamics (North-Holland, Amsterdam, 1974).

[3] R.I. Epstein, Ap. J. 333, 880 (1988).

[4] L. Lindblom \& G. Mendell, Ap. J. 421689 (1994).

[5] N. Andersson \& G.L. Comer, MNRAS 3281129 (2001).

[6] M.E. Gusakov \& N. Andersson, MNRAS 372, 1776 (2006).

[7] N. Andersson, G.L. Comer \& K. Glampedakis, Nucl. Phys. A 763, 212 (2005).

[8] M. Alford, A. Schmitt, K. Rajagopal \& T. Schäfer, Rev. Mod. Phys. 80, 1455 (2008).

[9] N. Andersson \& G. L. Comer, Entropy entrainment in finite temperature superfluids, preprint arXiv:0811.1660

[10] P. Jaikumar, G. Rupak \& A.W. Steiner, Phys. Rev. D 78123007 (2008).

[11] C. Manuel \& F.J. Llanes-Estrada, JCAP 8, 1 (2007).

[12] C. Manuel, A. Dobado \& F.J. Llanes-Estrada, JHEP 9, 76 (2005).

[13] M. Mannarelli, C. Manuel \& B.A. Sa'd, Phys. Rev. Lett. 101, 241101 (2008).

[14] M. Mannarelli \& C. Manuel, Phys. Rev. D 81, 043002 (2010).

[15] M.G. Alford, M. Braby, S. Reddy \& T Schäfer, Phys. Rev. C. 75 055209, (2007).

[16] M.G. Alford, M. Braby \& A. Schmitt, J. Phys. G. 35, 115007 (2008).

[17] M.G. Alford, M. Braby \& S. Mahmoodifar, Phys. Rev. C 81025202 (2010). 
[18] R. Prix, Phys. Rev. D 69043001 (2004).

[19] N. Andersson \& G.L. Comer, Class. Quantum Grav. 235505 (2006).

[20] N. Andersson \& G.L. Comer, Living Reviews in Relativity, 10 no. 1 (2007).

[21] N. Andersson \& G.L. Comer, Proc. R. Soc. London A, doi 10.1098/rspa.2009.0423

[22] N. Andersson, T. Sidery \& G.L. Comer, MNRAS 368, 162 (2006).

[23] C. Alcock. E. Farhi \& A. Olinto, Ap. J. 310, 261 (1986).

[24] N. Andersson \& K.D. Kokkotas, Int. J. Mod. Phys. D 10, 381 (2001).

[25] B. Haskell \& N. Andersson, Superfluid hyperon bulk viscosity and the r-mode instability of rotating neutron stars, preprint arXiv:1003.584

[26] K.H. Lockitch, N. Andersson \& J.L. Friedman, Phys. Rev. D 63, 024019 (2001).

[27] K.H. Lockitch, J.L. Friedman \& N. Andersson, Phys. Rev. D 68, 124010 (2003).

[28] J. Ruoff \& K.D. Kokkotas, MNRAS 328, 678 (2001).

[29] J. Ruoff \& K.D. Kokkotas, MNRAS 330, 1027 (2002).

[30] G.L. Comer, Found. Phys. 321903 (2002).

[31] N. Andersson, K. Glampedakis \& B. Haskell, Phys. Rev. D 79, 103009 (2009).

[32] B. Haskell, N. Andersson \& A. Passamonti, MNRAS 397, 1464 (2009).

[33] R. Prix \& M. Rieutord, Astron. Astrophys. 393, 949 (2002).

[34] A. Passamonti, B. Haskell \& N. Andersson, MNRAS 396, 951 (2009).

[35] B. Carter \& D. Langlois, Phys. Rev. D 515855 (1995).

[36] A.A. Zadorozhko, E. Ya. Rudavski, V.K. Chagovets, G.A. Sheshin \& Yu. A. Kitsenko, Low. Temp. Phys. 35, 100 (2009).

[37] J. Madsen, Phys. Rev. D 46, 3290 (1992).

[38] R.J. Donnelly, Quantized vortices in Helium II (Cambridge University Press, Cambridge, 1991).

[39] K. Glampedakis, N. Andersson \& D.I. Jones, MNRAS 394, 1908 (2009).

[40] L. Lindblom, B.J. Owen \& S.M. Morsink, Phys. Rev. Lett. 80, 4843 (1998)

[41] L. Lindblom \& G. Mendell, Phys. Rev. D 60, 064006 (1999).

[42] D.N. Aguilera, J.A. Pons, J.A. Miralles, Ap. J. Lett. 673, 167 (2008).

[43] E.H. Gudmundsson, C.J. Pethick \& R.I. Epstein, Ap. J. 272, 286 (1983).

[44] K. Glampedakis \& N. Andersson, MNRAS 371, 1311 (2006).

[45] K. Glampedakis \& N. Andersson, Phys. Rev. D 74, 044040 (2006).

[46] L. Bildsten, Ap. J. Lett. 501, 89 (1998).

[47] N. Andersson, K.D. Kokkotas \& N. Stergioulas, Ap. J. 516, 307 (1999).

[48] L. Lindblom \& G. Mendell, Ap. J. 444, 804 (1995).

[49] L. Lindblom \& G. Mendell, Phys. Rev. D 61, 104003 (2000).

[50] N. Andersson, D.I. Jones \& K.D. Kokkotas, MNRAS 337, 1224 (2002).

[51] Throughout this paper we use a coordinate basis to represent tensorial relations. That is, we distinguish between co- and contra-variant objects, $v_{i}$ and $v^{i}$, respectively. Indices, which range from 1 to 3 , can be raised and lowered with the (flat space) metric $g_{i j}$, i.e., $v_{i}=g_{i j} v^{j}$. Derivatives are expressed in terms of the covariant derivative $\nabla_{i}$ which is consistent with the metric in the sense that $\nabla_{i} g_{k l}=0$. This formulation has great advantage when we want to discuss the geometric nature of the different dissipation coefficients. 